\title{
Using urinary bFGF and TIMP3 levels to predict the presence of juvenile pilocytic astrocytoma and establish a distinct biomarker signature
}

\author{
Katie Pricola Fehnel, MD, ${ }^{1,2,4}$ Micah Duggins-Warf, BA, ${ }^{1,2}$ David Zurakowski, PhD, ${ }^{3}$ \\ Maxwell McKee-Proctor, BA,, Rajarshi Majumder, MS,, Michael Raber, MD,,2 \\ Xuezhe Han, MD, PhD, 1,2 and Edward R. Smith, MD ${ }^{1,2}$
} ${ }^{1}$ Vascular Biology Program, and Departments of ${ }^{2}$ Neurosurgery and ${ }^{3}$ Surgery, Boston Children's Hospital; and ${ }^{4}$ Department of
Neurosurgery, Massachusetts General Hospital, Boston, Massachusetts

\begin{abstract}
OBJECTIVE The authors report the use of urinary biomarkers as a novel, noninvasive technique to detect juvenile pilocytic astrocytomas (JPAs), capable of distinguishing JPAs from other CNS diseases, including other brain tumors. Preliminary screening of an array of tumors implicated proteases (including matrix metalloproteinases [MMPs]) and their inhibitors (tissue inhibitors of metalloproteinase [TIMPs]) as well as growth factors (including basic fibroblast growth factor [bFGF]) as candidate biomarkers. These data led the authors to hypothesize that tissue inhibitor of metalloproteinase 3 (TIMP3) and bFGF would represent high-probability candidates as JPA-specific biomarkers.
\end{abstract}

METHODS Urine was collected from 107 patients, which included children with JPA ( $n=21)$, medulloblastoma $(n=17)$, glioblastoma $(n=9)$, arteriovenous malformations $(n=25)$, moyamoya $(n=14)$, and age- and sex-matched controls $(n=$ 21). Biomarker levels were quantified with enzyme-linked immunosorbent assay, tumor tissue expression was confirmed with immunohistochemical analysis, and longitudinal biomarker expression was correlated with imaging. Results were subjected to univariate and multivariate statistical analyses.

RESULTS Using optimal urinary cutoff values of bFGF $>1.0 \mathrm{pg} / \mathrm{\mu g}$ and TIMP3 $>3.5 \mathrm{pg} / \mathrm{\mu g}$, multiplexing bFGF and TIMP3 predicts JPA presence with 98\% accuracy. Multiplexing bFGF and MMP13 distinguishes JPA from other brain tumor subtypes with up to $98 \%$ accuracy. Urinary biomarker expression correlated with both tumor immunohistochemistry and in vitro tumor levels. Urinary bFGF and TIMP3 decrease following successful tumor treatment and correlate with changes in tumor size.

CONCLUSIONS This study identifies 2 urinary biomarkers-bFGF and TIMP3 - that successfully detect one of the most common pediatric brain tumors with high accuracy. These data highlight potential benefits of urinary biomarkers and support their utility as diagnostic tools in the treatment of children with JPA.

http://thejns.org/doi/abs/10.3171/2015.12.PEDS15448

KEY WORDS pilocytic astrocytoma; basic fibroblast growth factor; bFGF; tissue inhibitor of metalloproteinase 3; TIMP3; urinary biomarkers; oncology

$\mathrm{J}$ UVENILE pilocytic astrocytomas (JPAs) are the most common pediatric brain tumors, with peak incidence in the first decade of life, accounting for approximately one-fifth of all brain tumors in patients younger than 14 years of age. ${ }^{6}$ These tumors are usually resected, but can recur even decades after treatment, mandating longi- tudinal follow-up. Subsequently, patients with JPA require long-term routine MRI studies. MRI is the current clinical "gold standard" for tumor detection, but can be expensive, necessitate inconvenient travel to dedicated centers, and often requires the risk of general anesthesia in children. In contrast, urinary biomarkers satisfy a need for an inex-

ABBREVIATIONS AUC = area under the curve; $\mathrm{AVM}=$ arteriovenous malformation; $b F G F=$ basic fibroblast growth factor; $\mathrm{Cl}=$ confidence interval; $\mathrm{DAPI}=4,6$ '-diamino2-phenylindole-dihydrochloride; DMEM = Dulbecco's modified Eagle's medium; EGF = epidermal growth factor; IQR = interquartile range; IRB = Institutional Review Board; JPA = juvenile pilocytic astrocytoma; MMP = matrix metalloproteinase; NF1 = neurofibromatosis Type 1; PBS = phosphate-buffered saline; ROC = receiver operating characteristic; TIMP3 = tissue inhibitor of metalloproteinase 3; VEGF = vascular endothelial growth factor.

ACCOMPANYING EDITORIAL See pp 393-395. DOI: 10.3171/2016.2.PEDS15724.

SUBMITTED July 22, 2015. ACCEPTED December 17, 2015.

INCLUDE WHEN CITING Published online June 17, 2016; DOI: 10.3171/2015.12.PEDS15448. 
pensive, noninvasive, universally accessible monitoring technique. ${ }^{7,20,27-32}$ We have previously demonstrated that urinary biomarkers can distinguish patients with CNS tumors accurately from age- and sex-matched controls. ${ }^{32}$ In this paper we describe the use of urinary biomarkers to detect a specific subtype of brain tumor (JPA).

Our biomarker species-tissue inhibitor of metalloproteinase 3 (TIMP3) and basic fibroblast growth factor (bFGF) - were selected based on previous work in our laboratory focused on extracellular proteases and angiogenic growth factors (which included these two molecules specifically) as markers of CNS disease, with evidence implicating them as critical to tumor development. bFGF plays an established role in the proliferation, differentiation, and migration of cells in CNS development, ${ }^{2,4,9,12,13,21}$ has been found to be overexpressed in adult brain tumors, ${ }^{25,35,36}$ and while not previously investigated specifically as a marker for pediatric brain tumors, has been successfully used as a urinary biomarker in both animal models and humans for more than 20 years. ${ }^{33}$

TIMP3 is one of the tissue inhibitors of matrix metalloproteinases (MMPs). In addition to our own laboratory experience implicating tissue inhibitors of metalloproteinase (TIMPs) and MMPs as critical regulators of tumor development, there is emerging literature specifically linking these molecules, including TIMP3, to brain tumors. Circulating levels of MMPs are elevated in high-grade gliomas and medulloblastoma, and TIMP3 expression is diminished in high-grade adult tumors. ${ }^{22,34}$ These reported studies, when coupled with the ongoing work in our laboratory, enabled us to significantly focus the panel of prospective biomarker candidates, with TIMP3 and bFGF ultimately emerging as the best predictors of JPA.

This work provides data that addresses defined clinical needs for one of the most common pediatric brain tumors, i.e., a novel method of tumor detection with noninvasive follow-up. In addition, this work presents the first brain tumor-specific urinary biomarker "fingerprint."

\section{Methods \\ Patient Population}

Specimens were collected in accordance with protocols approved by the Boston Children's Hospital Institutional Review Board (IRB), including prospective collection of urine from patients evaluated at Boston Children's Hospital. Informed consent was obtained. Inclusion criteria consisted of patients undergoing clinical evaluation at Boston Children's Hospital with MRI of the CNS. A total of 107 patients (Supplementary Table 1) were included in this study, of whom 21 had received the diagnosis of JPA. Patients with JPA included newly diagnosed, untreated JPA $(n=15)$ and patients with recurrent JPA $(n=6)$. No patient had measurable or documented evidence of metastasis. Control urine samples were collected from healthy ageand sex-matched patients $(n=21)$, all of whom underwent CNS imaging to document the absence of any neoplastic or vascular disease in the brain or spine. Additional patient cohorts obtained for comparative analysis included other pediatric tumors, such as medulloblastoma $(n=17)$ and glioblastoma $(n=9)$, as well as pediatric vascular dis- eases such as arteriovenous malformation (AVM; $n=25$ ) and moyamoya $(n=14)$. Treatment plans of the patients included in this study were not altered by or contingent upon this study; patients all received the current standard of care, which was attempted gross-total resection with subsequent need for chemotherapy with or without radiation, determined by a multidisciplinary care team at Boston Children's Hospital and the Dana Farber Cancer Institute (Boston, Massachusetts). In accordance with approved protocols, posttreatment specimens were collected between 4 and 72 months after resection in the follow-up brain tumor clinic $(n=9)$. This biomarker study is presented in accordance with reporting recommendations for tumor marker prognostic studies. ${ }^{18}$

\section{Urine Collection}

Urine was collected as part of an IRB-approved protocol between 2006 and 2014 and as previously reported by us. ${ }^{32}$ Urine samples were collected preoperatively, the same day as planned surgical treatment. No patient had evidence of major physiological stressors such as stroke, recent surgery, chemotherapy, or radiation at the time of collection. Immediately after collection, samples were transported on ice to the laboratory, stored at $-80^{\circ} \mathrm{C}$, and biomarker levels were assessed by Bio-Plex Multiplex System assays (Bio-Rad Laboratories) after determining the urine protein concentration by Bradford assay, as previously reported. . $^{31,32}$ Approximately 10-30 $\mathrm{ml}$ of urine was collected per patient collection, but only $100 \mu \mathrm{l}$ are needed for BioPlex analysis, with up to 6 putative biomarkers able to be measured concurrently with each 100- $\mu$ l sample. Excess urine is stored per protocol. Follow-up urine samples were collected more than 3 months postoperatively to control for confounders of normal wound healing. ${ }^{19}$

\section{Tissue Collection}

Tissue specimens were obtained from the Department of Pathology, Division of Neuropathology, at Boston Children's Hospital through the Dana Farber Cancer InstituteIntegrated Tissue and Clinical Data Bank for Patients with Neurological Disorders and in accordance with an IRBapproved protocol. Representative slides of brain tumor tissue were prepared from paraffin-embedded tissue for immunohistochemical staining. ${ }^{32}$

\section{Primary Tumor Cell Culture}

Primary cell cultures $(n=3)$ were derived by the following protocol and in collaboration with the Ligon laboratory at the Dana Farber Cancer Institute (Boston, Massachusetts). A tumor specimen was obtained from the surgical suite on ice and taken immediately to the sterile tissue culture hood. Tissue was then rinsed with sterile phosphate-buffered saline (PBS), dissected into $0.5-\mathrm{cm}$ sections, transferred to a falcon tube with Dulbecco's modified Eagle's medium (DMEM)/nutrient mixture F12, $10 \%$ fetal bovine serum, and penicillin/streptomycin antibiotic. Tissue was centrifuged for 5 minutes at $1000 \mathrm{~g}$, solute was carefully aspirated without disrupting cell/tissue suspension, and then resuspended in $0.25 \%$ trypsin in DMEM. This tissue/trypsin mixture was then placed in a 
$37^{\circ} \mathrm{C}$ water bath for 30 minutes with intermittent agitation and centrifuged for 5 minutes at $1000 \mathrm{~g}$. The solute was aspirated, the tissue was resuspended in media, and then plated in a T75 flask. Primary tumor cultures were expanded and tumor lineage was confirmed with a tumorspecific marker, Olig2. ${ }^{24}$ Conditioned media was collected from serum-starved cells after 24 hours and stored at $-80^{\circ} \mathrm{C}$

\section{Immunofluorescence}

Primary JPA cell cultures were fixed in $10 \%$ formalin and permabealized in $0.1 \%$ Triton X-100. Cells were then blocked in 5\% goat serum in PBS and stained with Olig2 (1:50; Sigma, Anti-Olig2 ALPA003254), bFGF (1:100; Abcam, ab8880), and TIMP3 (1:500; Abcam, ab39185) overnight at $4^{\circ} \mathrm{C}$. Cells were then incubated with Alexa Fluor 594 goat anti-rabbit secondary antibody (Life Technology), and counterstained with 4,6'-diamino-2-phenylindole-dihydrochloride (DAPI). Under magnification of 200, JPA Olig2 staining was compared with negative control human umbilical vein endothelial cells, ${ }^{24}$ and JPA bFGF and TIMP3 staining was compared with human adult astrocytes.

\section{Urinary and Tissue Biomarker Analysis}

\section{Bio-Plex Multiplex System Assays}

Biomarker profiling was performed on all patient urine samples and cell-line conditioned media in duplicate. Custom Fluorokine Multianalyte Profiling polystyrene bead assays (R\&D Systems) were designed to test MMP2, MMP3, MMP9, and MMP13, vascular endothelial growth factor (VEGF), epidermal growth factor (EGF), bFGF, heparin-binding EGF-like growth factor, and hepatic growth factor (R\&D Systems). TIMP profiling was performed utilizing a premixed Fluorokine Multianalyte Profiling Multiplex Human TIMP 4-plex kit (R\&D Systems). Assays were performed per company protocol.

\section{Immunohistochemical Analysis}

Representative tumor sections were obtained and confirmed by neuropathology. Ten-micrometer sections were cut from paraffin-embedded blocks for each patient specified. Tissue was deparaffinized in xylene and rehydrated in a graded ethanol series (100\% for 5 minutes, $90 \%$ for 3 minutes, $80 \%$ for 3 minutes, and $50 \%$ for 3 minutes), and then permeabilized with PBS and $0.05 \%$ Triton X-100 for 5 minutes. Heat-mediated antigen retrieval was performed at $100^{\circ} \mathrm{C}$ in a microwave for 20 minutes using $10 \mathrm{mM}$ trisodium citrate and $0.05 \%$ Tween-20. Tissue was then blocked at room temperature in $10 \%$ goat serum in PBS, followed by primary incubation at $4^{\circ} \mathrm{C}$ overnight. Immunohistochemical analysis of tumor specimens was performed using antibodies to bFGF (1:100; Abcam, ab8880) and TIMP3 (1:150; Abcam, ab39185). Slides were then washed with PBS and $0.05 \%$ Triton X-100. Endogenous peroxidase blocking was performed in $0.3 \% \mathrm{H}_{2} \mathrm{O}_{2}$ in $\mathrm{PBS}$ and slides were incubated in horseradish peroxidase-conjugated secondary antibody. After washing, slides were treated with diaminobenzidine and hematoxylin counterstain, and then rehydrated. Staining was quantified us- ing Image J software (NIH). For each patient, 25 separate fields were reviewed at a magnification of 200; total cell number and total number of cells demonstrating immunoreactivity were counted and the percentage positive was calculated for each image. These 25 fields were then averaged to obtain the overall percentage of cells demonstrating immunoreactivity for a given protein species in each patient.

\section{MRI-Based Calculation of Tumor Area}

For all patients included, tumor imaging characteristics were assessed by postcontrast MRI. The area of enhancing tumor was calculated using the Macdonald glial tumor response criteria, which measure the largest crosssectional diameter by the largest cross-sectional diameter perpendicular to it. ${ }^{17}$ Tumor size was correlated with urinary biomarker levels.

\section{Longitudinal Follow-up}

JPA-specific urinary biomarkers were assessed for potential clinical utility in interval monitoring for disease progression and recurrence; individual patient responses to treatment were analyzed. Posttreatment biomarker response was evaluated by measuring urinary levels of TIMP3 and bFGF in patients $(\mathrm{n}=9)$ for whom preoperative and postoperative urine specimens had been obtained and compared with changes in imaging.

Patients had postoperative urine samples collected more than 3 months postoperatively to allow for normal wound healing to occur (range 4-72 months). ${ }^{32}$ Pre- and posttreatment tumor imaging characteristics were assessed by postcontrast MRI. The area of enhancing tumor was calculated using the Macdonald glial tumor response criteria (which measure the largest cross-sectional diameter by the largest cross-sectional diameter perpendicular to it) and changes in tumor size were compared with changes in urinary biomarker levels. ${ }^{17}$

\section{Statistical Analysis}

Power analysis indicated that the sample sizes of 21 patients with JPAs, 21 controls, and 17 patients with medulloblastoma provided $80 \%$ power to detect 2 -fold differences in median levels of the urinary biomarkers using the nonparametric Mann-Whitney U-test with a 2-sided $\alpha$-level of 0.05 (nQuery Advisor, version 7.0, Statistical Solutions). Box-and-whisker plots were used to represent median and interquartile ranges (IQRs) for each biomarker. ${ }^{3}$ Receiver operating characteristic (ROC) curve analysis was applied to assess the predictive accuracy of the biomarkers with area under the curve (AUC) and 95\% confidence intervals (CIs) as indices of diagnostic performance in differentiating between JPA and controls as well as JPA and medulloblastoma..$^{14}$ Multivariable logistic regression analysis was performed to determine the independent predictive biomarkers for differentiating between patients with JPA and controls and JPA versus medulloblastoma using the likelihood ratio test to assess significance..$^{15}$ Youden's index in ROC analysis was used to identify the optimal cutoff values (pg/ug) for bFGF, TIMP3, and MMP13, and based on these cutoff values, sensitivity, specificity, and accuracy 
were calculated. ${ }^{37}$ Statistical analysis was conducted using IBM/SPSS software (version 21.0, IBM). Two-tailed values of $p<0.05$ were considered statistically significant. As has been previously established and reported in the literature, ${ }^{32}$ dual marker multiplexing was performed to enhance mathematical validity and diagnostic certainty.

\section{Results}

\section{Baseline Characteristics of Patient Population}

A total of 107 patients were included in the study, of whom 21 had JPA. There were no dropouts, as the study focused on patients at the time of diagnosis. Longitudinal follow-up is ongoing. All MR images were reviewed by neuroradiologists. A total of 86 other children were included in the study: 9 with glioblastoma, 17 with medulloblastoma, 25 with AVMs, 14 with moyamoya, and 21 matched control patients. All patient demographics are presented in Supplementary Table 1; focused demographics of control patients, patients with JPA, and patients with medulloblastoma are presented in Table 1. Of the patients with JPAs, 15 were newly diagnosed tumors with specimen collection at the time of initial surgery and 6 patients had recurrent tumors with specimen collection at the time of reoperation. Genetic screening for $B R A F$ mutations revealed that 5 patients had 3' BRAF duplication, 1 patient had a V600E mutation, and 2 patients had NF1 mutations. No patients were currently receiving chemotherapy or radiation at the time of urine collection, although 3 patients had previously undergone chemotherapy.

Univariate comparisons between tumor patients and control subjects did not show relevant differences by age or sex (Table 1, Supplementary Table 1). No control subjects had a documented history of tumors, vascular malformations, or recent surgery (defined as within 3 months of specimen collection). No patient with a tumor or any control patient was critically ill or receiving any adjuvant therapies at the time of sample collection.

\section{Bio-Plex Multiplex System Assays}

Levels of MMP2, MMP3, MMP9, MMP13, TIMP1, TIMP2, TIMP3, TIMP4, bFGF, VEGF, and EGF in urine were quantitated using commercially available Luminex assays (ThermoFisher). Results were normalized to overall urinary protein concentration and levels from patients with JPAs were compared with levels from control patients. Subsequent analyses were performed to compare biomarker levels from patients with JPAs with biomarker levels in other pediatric CNS disease, including tumors (medulloblastoma and glioblastoma) and vascular disease (AVM and moyamoya). Statistically significant differences in biomarker expression were identified for 3 molecules following multivariate analysis: TIMP3, MMP13, and bFGF (Supplementary Table 1). Focus was given to biomarker levels in controls, patients with JPAs, and patients with medulloblastoma (Table 1).

\section{bFGF and TIMP3 Distinguish JPA From Controls}

Multiple stepwise logistic regression analysis revealed that, independent of age and sex, urinary bFGF and TIMP3 demonstrated robust statistical significance in distinguishing between patients with JPAs and controls (Fig. 1). These biomarkers were similarly unique to patients with JPAs as compared with other distinct CNS disease cohorts (Table 1). Median levels of urinary bFGF were $2.3 \mathrm{pg} / \mu \mathrm{g}$ (IQR 1.1-4.9) for patients with JPA and $0.16 \mathrm{pg} /$ $\mu \mathrm{g}$ (IQR 0.11-0.75) for controls; median levels of urinary TIMP3 were $5.1 \mathrm{pg} / \mu \mathrm{g}$ (IQR 3.3-11.1) for JPA and 0.92 $\mathrm{pg} / \mu \mathrm{g}$ (IQR 0.86-3.14) for controls (Fig. 1A).

Regression modeling demonstrated that a urinary bFGF value $>1.0 \mathrm{pg} / \mu \mathrm{g}$ has a 6 -fold increased likelihood of JPA presence and a urinary TIMP3 value $>3.5 \mathrm{pg} / \mu \mathrm{g}$ has an 11-fold increased likelihood of JPA presence versus controls, independent of age and sex (Fig. 1B and C). ROC analysis was performed to determine optimal cutoff points for both biomarkers, which were $1.0 \mathrm{pg} / \mu \mathrm{g}$ for

TABLE 1. Demographics and univariate comparisons of urinary biomarkers between the study groups*

\begin{tabular}{cccc}
\hline Variable & JPA & \multicolumn{1}{c}{ Controls } & Medulloblastoma \\
\hline No. of patients & 21 & 21 & 17 \\
\hline Mean age in yrs \pm SD & $9.2 \pm 5.9$ & $7.1 \pm 4.4$ & $8.4 \pm 4.5$ \\
\hline Sex (\%) & & & \\
\hline Female & $9(43)$ & $14(67)$ & $10(59)$ \\
\hline Male & $12(57)$ & $7(33)$ & $7(41)$ \\
\hline TIMP3 $(\mathrm{pg} / \mathrm{\mu g}) \dagger$ & $5.1(3.3-11.1)$ & $0.92(0.86-3.14) \ddagger$ & $10.6(4.9-18.4)$ \\
\hline MMP13 $(\mathrm{pg} / \mathrm{\mu g}) \dagger$ & $0.0(0.0-0.0)$ & $0.38(0.13-1.02) \S$ & $1.81(1.48-3.00) \ddagger$ \\
\hline bFGF $(\mathrm{pg} / \mathrm{\mu g}) \dagger$ & $2.3(1.1-4.9)$ & $0.16(0.11-0.75) \ddagger$ & $0.36(0.16-0.70) \ddagger$ \\
\hline
\end{tabular}

* Comparative demographics for all study patients reveal no statistically significant differences in age or sex. The table shows summarized univariate analysis highlighting JPA-specific biomarkers, with data supporting a JPA biomarker "fingerprint" as compared to other CNS diseases (including other tumor types). By univariate analysis, urinary bFGF shows statistically significant increases in patients with JPA versus matched controls as well as versus each of the investigated CNS diseases, including medulloblastoma, glioblastoma, AVM, and moyamoya. Urinary levels of TIMP3 show statistically significant differences between patients with JPAs and matched controls, patients with glioblastoma, and patients with AVM. MMP13 is notably absent in patients with JPAs versus both control and disease populations, most significantly when compared to elevated levels of MMP13 in medulloblastoma.

$\dagger$ Biomarker data are summarized using medians (IQRs) with the nonparametric Mann-Whitney U-test used to compare each study group to JPA.

$\ddagger p<0.001$.

$\S p<0.01$. 

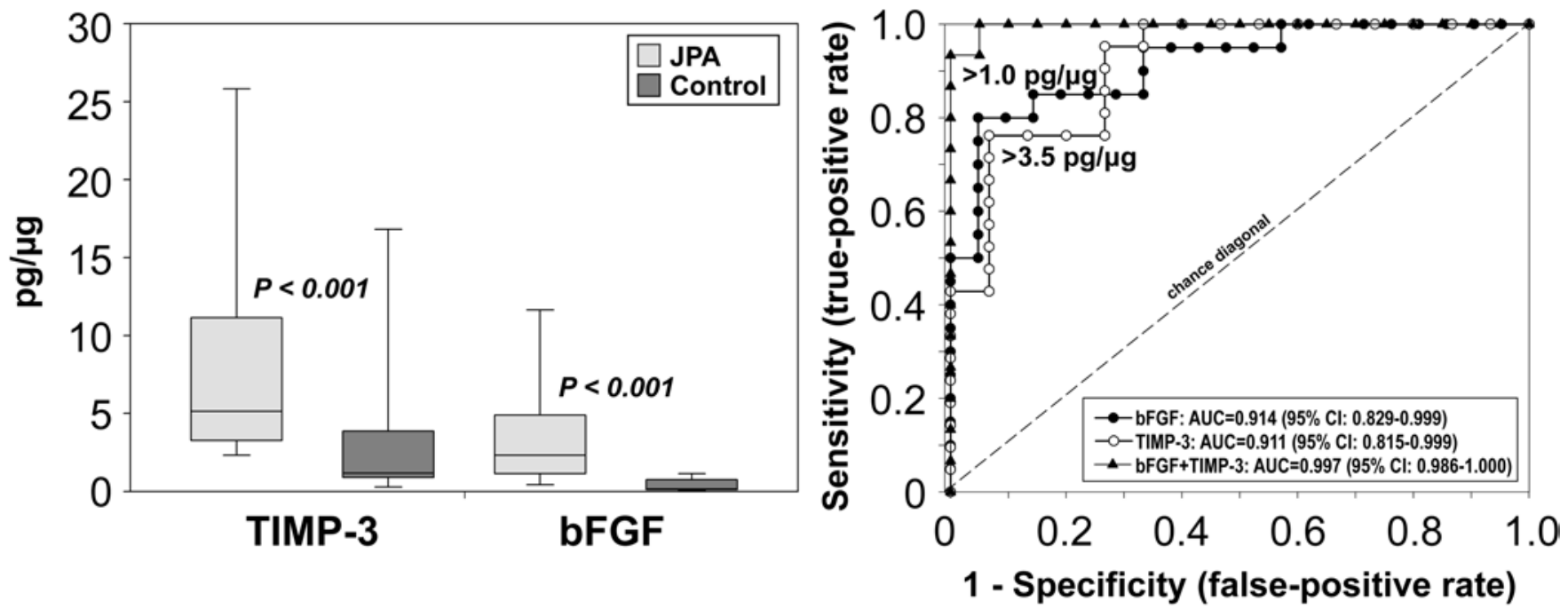

C

Diagnostic Performance of Urinary Biomarkers in Differentiating JPA and Control

\begin{tabular}{|c|c|c|c|c|c|c|}
\hline $\begin{array}{l}\text { Biomarker } \\
(\mathrm{pg} / \mu \mathrm{g})\end{array}$ & AUC (95\%) & $P$ value & $\begin{array}{l}\text { Optimal } \\
\text { cut-off }\end{array}$ & Sensitivity & Specificity & Accuracy \\
\hline TIMP-3 & $0.911(0.815-0.999)$ & $<0.001$ & $>3.5 \mathrm{pg} / \mu \mathrm{g}$ & $77 \%$ & $93 \%$ & $83 \%$ \\
\hline bFGF & $0.914(0.829-0.999)$ & $<0.001$ & $>1.0 \mathrm{pg} / \mathrm{\mu g}$ & $85 \%$ & $86 \%$ & $85 \%$ \\
\hline Both & $0.997(0.986-1.000)$ & $<0.001$ & Same & $100 \%$ & $95 \%$ & $98 \%$ \\
\hline
\end{tabular}

FIG. 1. A: Urinary levels of bFGF and TIMP3 are capable of distinguishing patients with JPA from healthy matched controls ( $p$ $<0.001$ ). Median levels of urinary bFGF were $2.3 \mathrm{pg} / \mu \mathrm{g}$ (IQR 1.1-4.9) for patients with JPA and $0.16 \mathrm{pg} / \mu \mathrm{g}$ (IQR 0.11-0.75) for controls; median levels of urinary TIMP3 were $5.1 \mathrm{pg} / \mathrm{\mu g}$ (IQR 3.3-11.1) for JPA and $0.92 \mathrm{pg} / \mathrm{\mu g}$ (IQR 0.86-3.14) for controls. Note that there was no overlap between the lowest levels of biomarkers from patients with tumors and the highest levels of biomarkers from control patients ( $n=21 \mathrm{JPAs}, n=21$ controls). B: Regression modeling demonstrated urinary biomarkers can predict the presence of tumor with high specificity and sensitivity. A urinary bFGF value $>1.0 \mathrm{pg} / \mu \mathrm{g}$ was 6 times more likely to indicate the presence of JPA (AUC 0.914 ) and a urinary TIMP3 value $>3.5 \mathrm{pg} / \mathrm{\mu g}$ was 11 times more likely to indicate the presence of JPA from controls (AUC 0.911), independent of age and sex. Multiplexed bFGF and TIMP3 are more than 20 times more likely to indicate JPA from controls (AUC 0.997). C: ROC analysis of urinary bFGF and TIMP3 shows excellent discrimination when used as independent markers for JPA versus controls, and $98 \%$ accuracy when multiplexed.

$\mathrm{bFGF}$ and $3.5 \mathrm{pg} / \mu \mathrm{g}$ for TIMP3. The AUC for bFGF was 0.914 (95\% CI 0.829-0.999) and for TIMP3 was 0.911 (95\% CI 0.815-0.999), both of which indicate excellent discrimination.

\footnotetext{
Multiplexing Biomarkers Markedly Enhances Diagnostic Accuracy

Multiplexing is an established and accepted biomarker technique that enhances the mathematical validity and diagnostic certainty. Multiplexing bFGF and TIMP3 together significantly increases the accuracy of distinguishing JPA from controls than with either biomarker alone $(\mathrm{p}<0.001)$. Using a bFGF value $>1.0$ and a urinary TIMP3 value $>$ $3.5 \mathrm{pg} / \mu \mathrm{g}$ is 20 times more likely to distinguish JPA from controls. The AUC for bFGF+TIMP3 was 0.997 (95\% CI $0.986-1.0)$ and a sensitivity of $100 \%$, a specificity of $95 \%$, and an overall accuracy of $98 \%$ (Fig. 1C).
}

\section{bFGF and MMP13 Distinguish JPA From Medulloblastoma}

The ability of urinary biomarkers to discriminate between subtypes of brain tumors was also tested. Specific analysis of medulloblastoma was performed in detail due to its prevalence (see Discussion). bFGF and MMP13 proved to be independent, highly selective markers capable of distinguishing JPA from medulloblastoma (Fig. 2). Median levels of urinary bFGF were elevated in patients with JPA (2.3 pg/ug [IQR 1.1-4.9]) versus patients with medulloblastoma $(0.36 \mathrm{pg} / \mu \mathrm{g}$ [IQR $0.16-0.70]$ for medulloblastoma). In contrast, median levels of urinary MMP13 were lower in patients with JPA compared with patients with medulloblastoma (0 pg/ug [IQR 0-0] for JPA and $1.8 \mathrm{pg} / \mu \mathrm{g}$ [IQR 1.48-3.00] for medulloblastoma; Fig. 2A).

Results of the regression modeling showed that a urinary bFGF value $>1.0 \mathrm{pg} / \mu \mathrm{g}$ is 7 times more likely to in- 

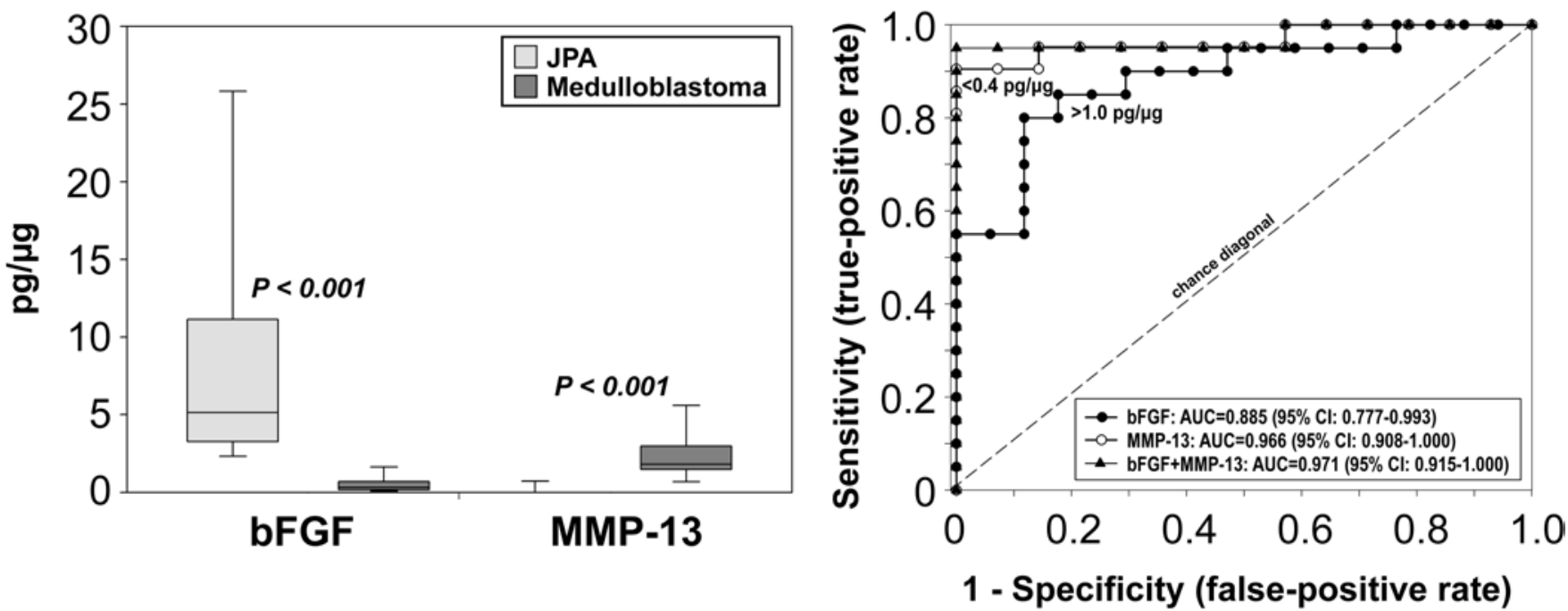

Diagnostic Performance of Urinary Biomarkers in Differentiating JPA \& Medulloblastoma

\begin{tabular}{|llrlrrc|}
\hline $\begin{array}{l}\text { Biomarker } \\
\text { (pg/ } / \mathbf{\mu g})\end{array}$ & AUC (95\%) & $\boldsymbol{P}$ value & $\begin{array}{l}\text { Optimal } \\
\text { cut-off }\end{array}$ & Sensitivity & Specificity & Accuracy \\
\hline MMP-13 & $0.966(0.908-1.000)$ & $<0.001$ & $<0.4 \mathrm{pg} / \mathrm{\mu g}$ & $91 \%$ & $100 \%$ & $95 \%$ \\
bFGF & $0.885(0.777-0.993)$ & $<0.001$ & $>1.0 \mathrm{pg} / \mathrm{\mu g}$ & $85 \%$ & $88 \%$ & $86 \%$ \\
Both & $\mathbf{0 . 9 7 1 ( 0 . 9 1 5 - 1 . 0 0 0 )}$ & $<0.001$ & Same & $\mathbf{9 5 \%}$ & $\mathbf{1 0 0 \%}$ & $\mathbf{9 8 \%}$ \\
\hline
\end{tabular}

FIG. 2. A: Example of the ability of urinary biomarkers to discriminate between different types of CNS tumors. By univariate analysis, levels of urinary bFGF and MMP13 can distinguish between patients with JPA and medulloblastoma $(p<0.001)$. Median levels of urinary bFGF were $2.3 \mathrm{pg} / \mathrm{\mu g}$ (IQR 1.1-4.9) for patients with JPA and $0.36 \mathrm{pg} / \mathrm{\mu g}$ (IQR 0.16-0.70) for medulloblastoma; median levels of urinary MMP13 were $0 \mathrm{pg} / \mathrm{\mu g}(\mathrm{IQR} 0-0)$ for JPA and $1.8 \mathrm{pg} / \mathrm{\mu g}$ (IQR 1.48-3.00) for medulloblastoma. B: Results of the regression modeling showed that a urinary bFGF value $>1.0 \mathrm{pg} / \mu \mathrm{g}$ was 7 times more likely to indicate JPA presence (AUC 0.885) and a urinary MMP13 value $<0.4 \mathrm{pg} / \mathrm{\mu g}$ was infinitely more likely to differentiate JPA from controls (AUC 0.966). Multiplexed bFGF and MMP13 revealed $100 \%$ sensitivity to distinguish JPA from medulloblastoma (AUC 0.971; $n=21 \mathrm{JPA}, n=17$ medulloblastoma). C: ROC analysis of bFGF and MMP13 showed excellent discrimination when used as independent markers for JPA versus medulloblastoma, and $98 \%$ accuracy when multiplexed ( $n=21$ JPA, $n=21$ controls).

dicate JPA presence and a urinary MMP13 value $<0.4 \mathrm{pg} /$ $\mu \mathrm{g}$ is infinitely more likely distinguish JPA from medulloblastoma, independent of age and sex (Fig. 2B and C). ROC analysis was performed to determine optimal cutoff points for both biomarkers, which was $1.0 \mathrm{pg} / \mu \mathrm{g}$ for $\mathrm{bFGF}$ and $0.4 \mathrm{pg} / \mu \mathrm{g}$ for MMP13. The AUC for bFGF was 0.885 (95\% CI 0.777-0.993) and the AUC for MMP13 was 0.966 (95\% CI 0.908-1.000).

Multiplexing the combination of bFGF and MMP13 together increased the accuracy of distinguishing JPA from medulloblastoma than with either biomarker alone $(\mathrm{p}<$ 0.001). The AUC for bFGF and MMP13 in combination was $0.971(95 \%$ CI $0.915-1.0)$ with a sensitivity of $95 \%$, specificity of $100 \%$, and an overall accuracy of $98 \%$ (Fig. 2C).

\section{Immunohistochemistry}

Urinary biomarker levels were correlated with tumor tissue expression by immunohistochemical analysis. Individual tumor specimens with a diagnosis of pilocytic astrocytoma $(n=5)$ were obtained from pathology and subjected to immunohistochemical analysis. bFGF and TIMP3 were detected in JPA tissue at significantly higher rates than in normal cerebellum $(n=2)$. Staining for $b F G F$ in JPA showed a 5.2-fold increase over normal cerebellum control $(\mathrm{p}=0.011)$, and staining for TIMP3 in JPA showed a 2.25-fold increase over normal cerebellum control $(\mathrm{p}=$ 0.003). These data indicate that JPA tumor tissue exhibits increased biomarker protein expression relative to normal brain (Fig. 3A and B).

\section{In Vitro Validation of Biomarkers}

Based on the immunohistochemical results, the urinary increase in bFGF and TIMP3 in JPAs was attributed to increased expression at the level of the tumor (Fig. 3). 
To further establish that bFGF and TIMP3 urinary levels are increased in patients with JPA due to production of protein from the tumor cells themselves, primary patientderived JPA tumor cell lines were established. Cell staining with a tumor-specific marker (Olig2) confirmed tumor lineage (Fig. 3C) ${ }^{24}$ Primary cultures were then evaluated by immunofluorescence for both bFGF and TIMP3. JPA cells highly expressed both bFGF (localized to the cell nuclei) and TIMP3 (localized to the cytoplasm; Fig. 3C). Additional tumor types have been similarly investigated (including glioblastoma, medulloblastoma, and ependymoma), and while many tumor lines express high levels of

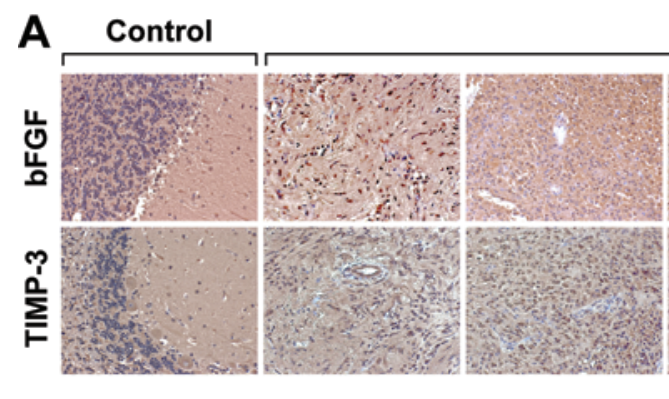

B

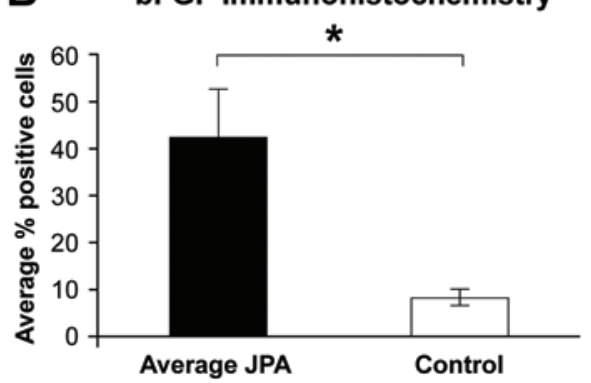

JPA

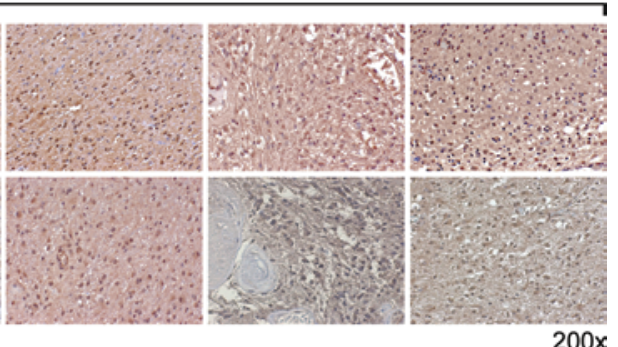

TIMP-3 Immunohistochemistry

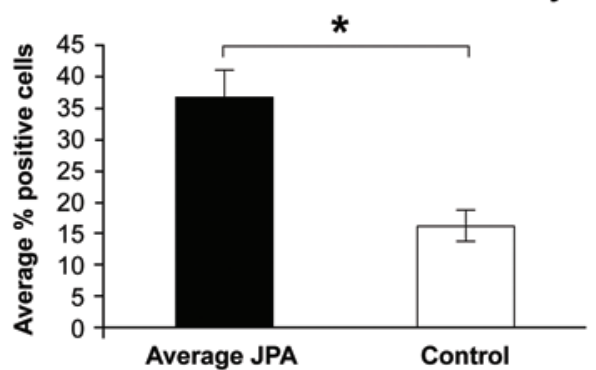

ii

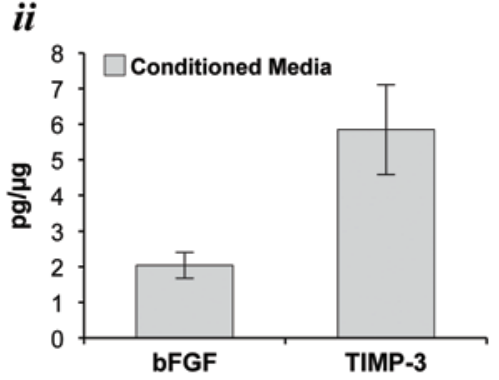

FIG. 3. A: Representative photomicrographs demonstrate tumor as confirmed by neuropathology. Immunohistochemical analysis identifies tumor tissue as a putative source of the urinary biomarker levels. bFGF, which localized predominantly to the nucleus, and TIMP3, which localized predominantly to the cytoplasm and cell membrane, were both detected in JPA tissue at significantly higher rates than in normal cerebellum. Diaminobenzidine and hematoxylin counterstain, original magnification $\times 200$. B: Number of cells with identifiable staining for bFGF showed a 5.2-fold increase over normal cerebellum control $(p=0.011)$ and staining for TIMP3 showed a 2.25-fold increase over normal cerebellum control $(p=0.003)$. C: Using JPA primary culture cell lines derived from 3 of the patients in our study, we demonstrated expression of the biomarker species directly from the source tissue. These data support the hypothesis that the tumor cells are a primary source of the biomarkers ultimately measured in the urine. In vitro bFGF expression most strongly localizes to the nucleus (bFGF = red stain, DAPI nucleus = blue stain), while TIMP3 can be seen both in the cytoplasm and cell membrane of primary JPA-derived cells (TIMP3 = red stain). Cells are confirmed as JPA tumor by Olig2-specific staining $(i)$. Original magnification $\times 200$. Average levels of in vitro secreted protein expression exceed the cutoff values for bFGF $(>1.0 \mathrm{pg} / \mathrm{ug})$ and TIMP3 $(>3.5 \mathrm{pg} / \mathrm{ug})$ as measured in conditioned media from patient-derived primary cell lines (n $=3)$ : bFGF level $=2.04 \mathrm{pg} / \mathrm{ug}$, TIMP3 level $=5.85 \mathrm{pg} / \mathrm{ug}(\mathrm{ii})$. 
bFGF, the combination of high levels of bFGF and TIMP3 expression are unique to JPA.

Conditioned media was then collected from serumstarved cells to evaluate for biomarker expression. Results showed average levels of cell-secreted protein, which exceeded our established urinary cutoff values (bFGF $>1.0$ and TIMP3 > 3.5): the bFGF level was $2.04 \mathrm{pg} / \mu \mathrm{g}$ (conditioned media) and TIMP3 level was $5.85 \mathrm{pg} / \mathrm{\mu g}$ (conditioned media; Fig. 3C). These data demonstrate that JPA tumor cells express biomarker proteins and that secreted levels of biomarkers in vitro recapitulate the levels measured in clinical practice from urine samples, supporting the premise that tumor cells are the source of the biomarker species found in the urine.

\section{Longitudinal Follow-Up}

After treatment, the mean area of residual enhancing tumor measured less than one-third of the original tumor area (1.9 vs $6.3 \mathrm{~cm}^{2}, \mathrm{p}=0.013$; Fig. 4A). Consistent with this data, mean posttreatment levels of urinary bFGF were less than one-third of the levels at the time of diagnosis, and urinary TIMP3 levels were less than one-half of the levels at the time of diagnosis. The average posttreatment urinary bFGF level was 2.8 versus $9.3 \mathrm{pg} / \mu \mathrm{g}$ before treatment ( $\mathrm{p}$ $=0.019$ ), and the average posttreatment TIMP3 level was 4.8 versus $10.8 \mathrm{pg} / \mu \mathrm{g}$ before treatment $(\mathrm{p}=0.040$; Fig. 4B). Representative patients are depicted in Fig. 4C and D.

\section{Correlation of Urinary Biomarker Levels With Current Diagnostic Standard (MRI)}

To validate urinary biomarker data against the standard prognostic variable (MRI), patients with pathologically proven JPA who were included in this analysis had biomarker levels paired with their preoperative imaging. Levels of bFGF and TIMP3 were not significantly higher in patients with larger tumors. Comparing smaller tumors measuring $0-10 \mathrm{~cm}^{2}(\mathrm{n}=10)$ with larger tumors measuring $10-25 \mathrm{~cm}^{2}(\mathrm{n}=10)$ showed no statistical trend with regard to bFGF $(p=0.83)$ or TIMP3 $(p=0.62)$. These data do not show a directly predictive correlation between absolute levels of biomarker and tumor size; rather, the data do show that trending individual patient-specific biomarker levels from time of diagnosis throughout treatment does correlate with tumor size. Changes in biomarker levels relative to each patient's initial baseline measurement serve as the best method to benchmark longitudinal follow-up (Fig. 4).

\section{Discussion}

Urinary biomarkers represent a novel, noninvasive diagnostic tool for CNS disease particularly applicable to pediatric brain tumors. Urine-based testing could substantially complement the current practice of serial imaging with MRI with its uniquely appealing attributes. Sampling can be conducted frequently, is risk-free (unlike sedation for MRI required in many children), cost-effective (more than 100 times less expensive than MRI at our institution), can be performed remotely and mailed (as is done already as part of our multicenter national trial of urinary brain tumor biomarkers [clinicaltrials.gov no. NCT01514201]) and represents a biologically discrete method of tumor diagnosis, distinct from the anatomical findings visualized on MRI.

Our laboratory has had significant experience in the discovery and validation of biomarkers of neurosurgical disease. We have previously shown that a panel of urinary biomarkers can identify the presence of brain tumors in adult and pediatric patients,,$^{32}$ that elevated urinary MMPs in brain tumor recurrence decrease following successful treatment, ${ }^{31}$ and that a specific urinary biomarker (netrin-1) can identify the presence of medulloblastoma with predictable treatment-specific responses. ${ }^{1}$ Importantly, we have also recently demonstrated that netrin-1 is a critical mediator of tumor invasion, thereby elucidating the biological relevance of netrin-1 as a biomarker and identifying it as a putative therapeutic target. ${ }^{1}$

However, to date there have not been studies of urinary biomarkers specific to JPA. Focus on this tumor subtype is important because it is the most common pediatric brain tumor and has the potential to progress or recur years later. As a consequence of this need for long-term follow-up, patients with JPA represent a cohort uniquely suited to benefit from the noninvasive, cheap, easily accessible screening capability offered by urinary biomarkers. ${ }^{6}$

\section{Selection of Biomarker Species}

Historically, bFGF was one of the first described proangiogenic factors ${ }^{11}$ and was noted to be elevated in the urine of patients with bladder cancer as well as numerous other solid tumors, leukemias, and lymphomas. ${ }^{23}$ Although bFGF is not a secreted factor, in malignancy it has been isolated in the urine of both humans and animal models for more than 20 years. ${ }^{33}$ bFGF plays a role in the proliferation, differentiation, and migration of cells of the CNS in development. . $^{2,4,9,12,13,21}$ Interestingly, some evidence suggests that while bFGF may be proangiogenic, it may also exert an antitumor effect in the CNS by retarding proliferation of oligodendrocytes and tumor cells. ${ }^{5}$ Taken together with preliminary screens of samples from patients in our lab, these findings suggested bFGF as a potential focus of study in this research.

In adult astrocytomas, bFGF expression is elevated in tumor tissue, ${ }^{25,35,36}$ as well as in the CSF of patients with high-grade gliomas ${ }^{26}$ and plasma of a mixed population of glioma patients..$^{16}$ Although the role of bFGF has not been specifically addressed in the pediatric astrocytoma literature, it has been shown to play a role in tumor development and progression in other pediatric CNS cancers. Specifically, treatment of medulloblastoma cells with bFGF inhibits proliferation both in vivo and in vitro by blocking sonic hedgehog signaling. ${ }^{8,10}$ It is particularly intriguing to speculate that this reported effect may be related to our finding of very low levels of urinary bFGF in patients with medulloblastoma (Fig. 2).

TIMP3 is one of the tissue inhibitors of metalloproteinases, which interact with MMPs. We (and others) have reported elevations in circulating levels of MMPs in patients with high-grade gliomas and medulloblastoma, which makes the study of TIMPs particularly interesting when looking at a low-grade noninvasive glioma like JPA. Adding support to the premise that levels of urinary biomark- 

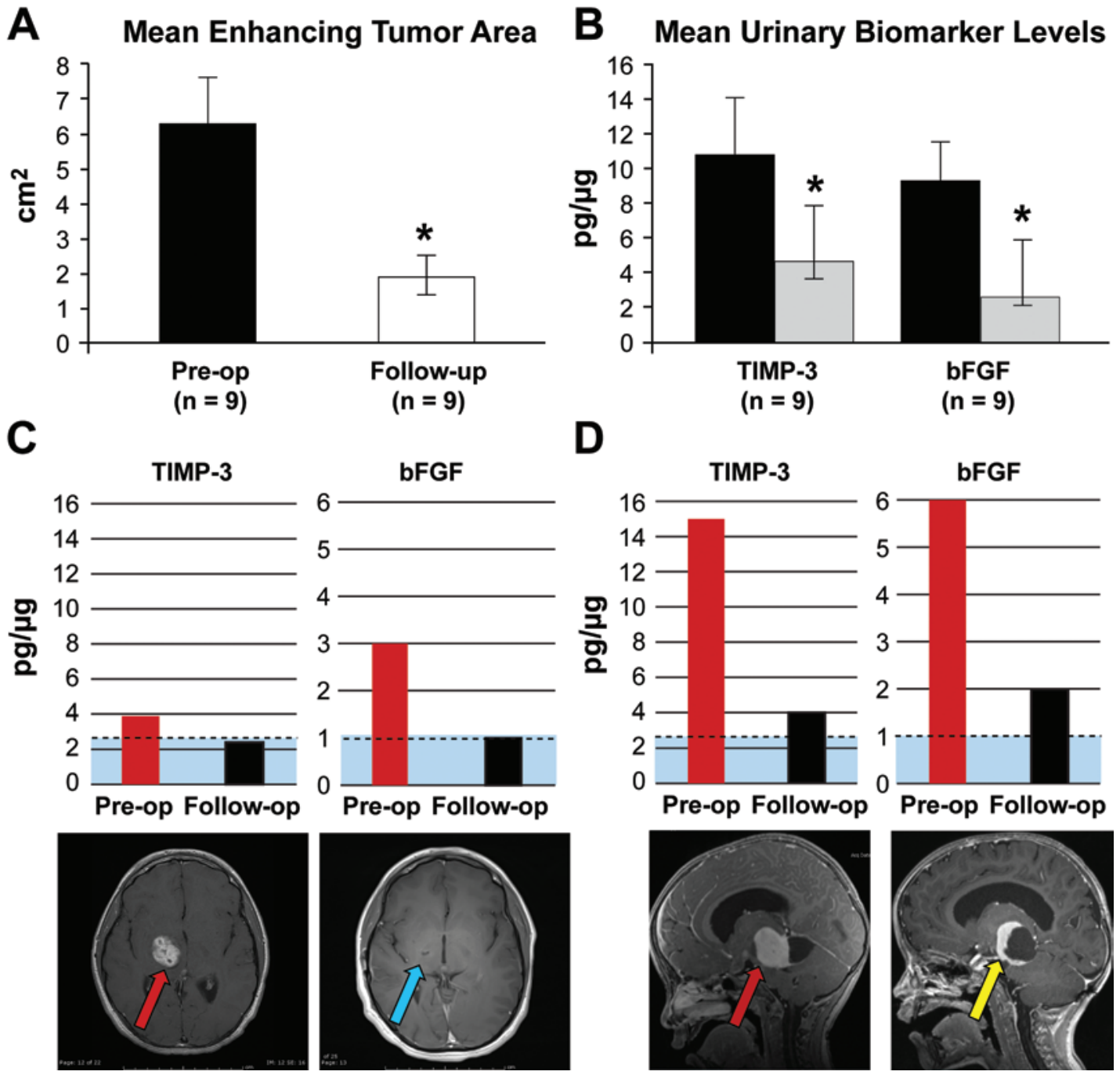

FIG. 4. Analysis of patients from whom preoperative and postoperative data were available demonstrates urinary biomarker changes concordant with radiographic outcome. In this cohort $(n=9)$, mean urinary levels of bFGF and TIMP3 decreased significantly following surgical treatment and correlated with radiographic evidence of reduction in enhancing tumor area. A: Radiographic follow-up data. On follow-up, postoperative MR images demonstrated an average reduction in tumor area to one-third of presentation area (6.3 vs $1.9 \mathrm{~cm}^{2} ; p=0.013$ ). B: Biomarker follow-up data. Mean posttreatment levels of bFGF (gray bars) were less than one-third of the levels at the time of diagnosis (black bars; $9.3 \mathrm{vs} 2.8 \mathrm{pg} / \mathrm{\mu g}, \mathrm{p}=0.019$ ). TIMP3 levels were more than halved posttreatment (10.8 vs $4.8, \mathrm{pg} / \mathrm{\mu g}, \mathrm{p}=0.040)$. C: Representative urinary biomarker and imaging data from a gross-total resection of a JPA. Graphs show preoperative urinary TIMP3 and bFGF levels, both of which are higher than their respective predicted tumor cutoff levels (dashed line, levels below tumor cutoff shaded blue area). The red arrow indicates the preoperative tumor. Following resection, urine biomarker levels drop below predicted cutoff levels, concordant with 1-year follow-up MRI showing complete resection without recurrence (blue arrow). D: Representative urinary biomarker and imaging data from a subtotal resection of a JPA. In this patient, preoperative urinary levels of TIMP3 and bFGF are elevated in the presence of tumor (red arrow), and drop at 1 year postoperatively, but remain above predicted tumor cutoff levels, concordant with the imaging data revealing residual tumor (yellow arrow).

ers accurately reflect patterns of molecular expression at the tumor site, elevated levels of TIMP3 have been noted in medulloblastoma tissue, and this finding-reported by others-directly correlates with our new data, showing high levels of urinary TIMP3 in patients with medulloblastoma (Table 1). ${ }^{22}$ Conversely, serum TIMP3 levels have been reported as decreased in patients with glioblastoma compared with controls. ${ }^{34}$ These findings in the serum are concordant with the low urinary levels we observed in our glioblastoma population and contrasts with the higher levels we report in JPA (Table 1).

\section{Advantages of Using bFGF and TIMP3}

In patients with pathologically proven JPA, levels of urinary bFGF and TIMP3 are markedly and significantly elevated compared with matched, healthy controls. Importantly, the data demonstrate that multiplexing the two markers provides far greater sensitivity and specificity than either marker used individually. With optimal cutoff values of $>1.0 \mathrm{pg} / \mu \mathrm{g}$ for $\mathrm{bFGF}$ and $>3.5 \mathrm{pg} / \mu \mathrm{g}$ for TIMP3, tumors can be identified with $98 \%$ accuracy (Fig. 1). In addition, for the first time this work establishes the successful detection of JPA using a urine test. 
We demonstrated elevated expression of bFGF and TIMP3 in pathological tissue specimens by immunohistochemical analysis, suggesting the tumor is the source of biomarkers detected in the urine (Fig. 3A and B). We further reinforced the premise that the tumor cells produce the molecules used as biomarkers by creating patient-derived tumor cell lines, confirming tumor cell lineage by tumor-specific markers and then measuring biomarker expression in vitro. Our data reveals that the tumor cells not only express the biomarker species reproducibly but that the levels expressed in vitro closely reflect the levels detected in vivo with urine samples collected in the clinical setting. Together, these findings link the laboratory data from in vitro work directly with the clinical application in the wards (Fig. 3).

One potential limitation with study of a single biomarker species and its capacity to distinguish between a single disease entity and healthy controls is whether the biomarker is truly unique to the disease or simply indicative of a generalized physiological process, such as angiogenesis or inflammation. While some of our earlier efforts suggested that different brain tumors may have specific biomarker "fingerprints," 32 the research presented here is the first, to our knowledge, to identify a brain tumor-specific urinary fingerprint. Although an exhaustive study is outside the scope of this paper, we have selected a representative range of neurosurgical disorders, including ischemic disease (moyamoya), vascular anomalies (AVM), high-grade glioma (glioblastoma), and nonglial primary brain cancer (medulloblastoma) and compared them to JPA (Supplementary Table 1, Table 1). We have concurrently compiled a series of markers that identify JPA as distinct from the other CNS pathologies noted above and-importantlyestablish unique "fingerprints" for each of these disorders relative to the others. This work comprises an independent project on CNS biomarker signatures, but we have included some of the data here as relevant to JPA, with a particular focus on medulloblastoma to demonstrate proof of principle of the capacity of urinary biomarkers to discern even between subtypes of pediatric brain tumor. This is important because it validates the practice of hypothesis-driven biomarker selection, suggests that screening for specific disorders in medically complex populations may be possible (such as screening for JPA in at-risk populations [as in children with neurofibromatosis Type 1, NF1] with cost effective, easy urine sampling), and offers the potential of using urinary biomarkers to identify and track disease-specific therapeutic targets.

We included a more detailed focus on medulloblastoma versus JPA because they have many similarities, highlighting the impact of identifying unique molecular signatures with biomarkers. In addition to both being primary tumors of the CNS that predominantly occur in the posterior fossa, they represent two of the most common pediatric brain tumors and share the need for long-term follow-up. Using a urine test, we demonstrated $98 \%$ accuracy in distinguishing JPA from medulloblastoma by multiplexing bFGF and MMP13 (Fig. 2). We anticipate broadening this effort to a wide spectrum of neurosurgical disorders.

\section{Clinical Utility}

These data indicate that JPA can be detected as a dis- tinct entity from other CNS diseases using a urine test consisting of 3 biomarker species (TIMP3, MMP13, and bFGF) with an accuracy of $98 \%$. In addition to the benefit of specific, sensitive diagnosis, which is particularly useful in screening at-risk populations, our clinical studies illustrate the potential for using urinary biomarkers to aid in long-term follow-up of treated patients. Urinary biomarkers vary directly in response to extent of resection and correlate with imaging data (Fig. 4). Both of these applications (improved diagnosis and longitudinal followup) have immense clinical value and could impact practice immediately.

We would emphasize that this technique, as currently reported, is not sensitive or specific enough yet to be used as a screening tool in the general population. Moreover, in the majority of cases, the need for surgery will remain, independent of any biomarker data. However, there may be some limited utility in using this as an adjunct to MRI screening in carefully selected at-risk populations, such as individuals who were found to have small lesions incidentally identified on imaging taken for other reasons. In these cases, it may be that urinary biomarkers could help to further reassure physicians that a small lesion noted on MRI is more likely to be a JPA, adding to the evidence to support the clinical plan of observation. As sensitivity and specificity increase with larger population data, it may be that urinary biomarkers could be used as screening tools in select, at-risk populations (such as patients with NF1) to offer a noninvasive screen for JPA that would reduce the need for MRI. In addition to potential screening applications, the use of noninvasive biomarkers could also play a role in following JPA after treatment. While absolute TIMP3 and bFGF levels do not directly correlate with tumor size, we have preliminary data that levels drop following successful treatment and that each patient can use preoperative levels as a baseline to help predict individual-specific responses to treatment (Fig. 4). In gross-total resection, levels drop down below cutpoint levels, while in patients with residual tumors, their urinary levels drop lower than they were at presentation, but do not cross the threshold below the cutpoint, indicating that tumor remains but with less volume than preoperatively. While these findings need further validation with larger cohorts, the preliminary data are promising in suggesting that urinary biomarkers may have some utility as a tool to complement and potentially reduce (but not replace) postoperative imaging. Given that JPA is a slow-growing tumor that may require lifelong follow-up, tools such as urinary biomarkers that are cheap, easy, and allow for reductions in travel to MRI centers by mailing in samples may be particularly beneficial to minimizing the financial and clinical impact of monitoring a benign, chronic condition.

\section{Potential Limitations and Future Directions}

The findings of this study are promising but some potential limitations should be acknowledged. One consideration is the relatively small number of patients. Even in our large tumor referral centers, not all patients met entry criteria or were willing to participate, and at times we have been limited by the amount of specimen available for analysis. Nonetheless, it should be noted that the results 
demonstrated robust statistical significance across multiple analyses. An additional consideration is whether other CNS diseases may manifest similar biomarker fingerprints to JPA, limiting the applicability of this approach. This possibility is currently being actively investigated in our laboratory, but we have sought to address this concern here by presenting comparisons across a spectrum of neurosurgical disease. It is tempting to speculate that the higher statistical power achieved by multiplexing these two molecules (bFGF and TIMP) may have implications beyond simple mathematics, given the potential complementary biological functions of a growth factor and a regulator of angiogenesis in tumor development. This utility as a biomarker pair has prompted us to now initiate a new series of ongoing studies designed to investigate potential novel functional relationships between bFGF and TIMP3 in JPA and other brain tumors. Lastly, it should be noted that only a subset of patients had samples available for longitudinal follow-up. Increasing the number of samples from individual patients, as well as increasing the total number of patients, will improve the strength of this part of the study and is ongoing at our center.

\section{Conclusions}

The prevalence of JPA underscores the importance of advancing the capability of physicians to accurately diagnose and monitor treatment of children with this tumor. The work presented here is significant as the first demonstration of a brain tumor-specific urinary biomarker "fingerprint," an important finding relevant to expanding the utility of this technique. Correlation of urinary biomarker expression from source tissue is matched for the first time with in vitro analysis of patient-derived tumor lines, reinforcing the claim that the brain tumor is the source of the urinary molecules bFGF and TIMP3. Lastly, the high degree of accuracy attainable with these biomarkers, bolstered by detailed clinical follow-up data, underscores the immediate clinical utility of this approach, although there remains a need to validate this work in large population studies. Ultimately, we envision that use of urinary biomarkers will enhance the current methods of diagnosis, follow-up, and therapeutic management of children with brain tumors.

\section{Acknowledgments}

We would like to thank Kristen Johnson for preparation of the article and Marsha Moses, $\mathrm{PhD}$, for her review of this manuscript and for all of her research support. This study was funded by Voices Against Brain Cancer and the Christopher Fellows Brain Tumor Research Fund (Dr. Smith), and the Neurosurgery Research and Education Foundation and AANS/CNS Section of Pediatric Neurological Surgery Research Fellowship (Dr. Pricola Fehnel).

\section{References}

1. Akino T, Han X, Nakayama H, McNeish B, Zurakowski D, Mammoto A, et al: Netrin-1 promotes medulloblastoma cell invasiveness and angiogenesis, and demonstrates elevated expression in tumor tissue and urine of patients with pediatric medulloblastoma. Cancer Res 74:3716-3726, 2014

2. Alberta JA, Park SK, Mora J, Yuk D, Pawlitzky I, Iannarelli $\mathrm{P}$, et al: Sonic hedgehog is required during an early phase of oligodendrocyte development in mammalian brain. Mol Cell Neurosci 18:434-441, 2001

3. Altman DG: Practical Statistics for Medical Research. Boca Raton, FL: Chapman and Hall, 1991

4. Bansal R: Fibroblast growth factors and their receptors in oligodendrocyte development: implications for demyelination and remyelination. Dev Neurosci 24:35-46, 2002

5. Bansal R, Pfeiffer SE: FGF-2 converts mature oligodendrocytes to a novel phenotype. J Neurosci Res 50:215-228, 1997

6. CBTRUS: Statistical Report: Primary Brain and Central Nervous System Tumors Diagnosed in the United States in 2004-2008. Hinsdale, IL: Central Brain Tumor Registry of the United States, 2012 (http://www.cbtrus.org/2012NPCR-SEER/CBTRUS_Report_2004-2008_3-23-2012.pdf) [Accessed April 6, 2016]

7. Coticchia CM, Curatolo AS, Zurakowski D, Yang J, Daniels KE, Matulonis UA, et al: Urinary MMP-2 and MMP-9 predict the presence of ovarian cancer in women with normal CA125 levels. Gynecol Oncol 123:295-300, 2011

8. Duplan SM, Théorêt Y, Kenigsberg RL: Antitumor activity of fibroblast growth factors (FGFs) for medulloblastoma may correlate with FGF receptor expression and tumor variant. Clin Cancer Res 8:246-257, 2002

9. Dyer C, Blanc E, Hanisch A, Roehl H, Otto GW, Yu T, et al: A bi-modal function of Wnt signalling directs an FGF activity gradient to spatially regulate neuronal differentiation in the midbrain. Development 141:63-72, 2014

10. Emmenegger BA, Hwang EI, Moore C, Markant SL, Brun SN, Dutton JW, et al: Distinct roles for fibroblast growth factor signaling in cerebellar development and medulloblastoma. Oncogene 32:4181-4188, 2013

11. Folkman J, Klagsbrun M: Angiogenic factors. Science 235:442-447, 1987

12. Gritti A, Frölichsthal-Schoeller P, Galli R, Parati EA, Cova L, Pagano SF, et al: Epidermal and fibroblast growth factors behave as mitogenic regulators for a single multipotent stem cell-like population from the subventricular region of the adult mouse forebrain. J Neurosci 19:3287-3297, 1999

13. Gritti A, Parati EA, Cova L, Frolichsthal P, Galli R, Wanke E, et al: Multipotential stem cells from the adult mouse brain proliferate and self-renew in response to basic fibroblast growth factor. J Neurosci 16:1091-1100, 1996

14. Hanley JA, McNeil BJ: The meaning and use of the area under a receiver operating characteristic (ROC) curve. Radiology 143:29-36, 1982

15. Katz MH: Multivariable Analysis. A Practical Guide for Clinicians and Public Health Researchers, ed 3. New York: Cambridge University Press, 2011

16. Kurimoto M, Endo S, Hirashima Y, Nishijima M, Takaku A: Elevated plasma basic fibroblast growth factor in brain tumor patients. Neurol Med Chir (Tokyo) 36:865-869, 1996

17. Macdonald DR, Cascino TL, Schold SC Jr, Cairncross JG: Response criteria for phase II studies of supratentorial malignant glioma. J Clin Oncol 8:1277-1280, 1990

18. McShane LM, Altman DG, Sauerbrei W, Taube SE, Gion M, Clark GM: REporting recommendations for tumour MARKer prognostic studies (REMARK). Eur J Cancer 41:1690-1696, 2005

19. Moses MA, Marikovsky M, Harper JW, Vogt P, Eriksson E, Klagsbrun M, et al: Temporal study of the activity of matrix metalloproteinases and their endogenous inhibitors during wound healing. J Cell Biochem 60:379-386, 1996

20. Moses MA, Wiederschain D, Loughlin KR, Zurakowski D, Lamb CC, Freeman MR: Increased incidence of matrix metalloproteinases in urine of cancer patients. Cancer Res 58:1395-1399, 1998

21. Mudò G, Bonomo A, Di Liberto V, Frinchi M, Fuxe K, Belluardo N: The FGF-2/FGFRs neurotrophic system promotes 
neurogenesis in the adult brain. J Neural Transm (Vienna) 116:995-1005, 2009

22. Mühlisch J, Bajanowski T, Rickert CH, Roggendorf W, Würthwein G, Jürgens H, et al: Frequent but borderline methylation of p16 (INK4a) and TIMP3 in medulloblastoma and SPNET revealed by quantitative analyses. J Neurooncol 83:17-29, 2007

23. Nguyen M, Watanabe H, Budson AE, Richie JP, Hayes DF, Folkman J: Elevated levels of an angiogenic peptide, basic fibroblast growth factor, in the urine of patients with a wide spectrum of cancers. J Natl Cancer Inst 86:356-361, 1994

24. Otero JJ, Rowitch D, Vandenberg S: OLIG2 is differentially expressed in pediatric astrocytic and in ependymal neoplasms. J Neurooncol 104:423-438, 2011

25. Paulus W, Grothe C, Sensenbrenner M, Janet T, Baur I, Graf $\mathrm{M}$, et al: Localization of basic fibroblast growth factor, a mitogen and angiogenic factor, in human brain tumors. Acta Neuropathol 79:418-423, 1990

26. Peles E, Lidar Z, Simon AJ, Grossman R, Nass D, Ram Z: Angiogenic factors in the cerebrospinal fluid of patients with astrocytic brain tumors. Neurosurgery 55:562-568, 2004

27. Pories SE, Zurakowski D, Roy R, Lamb CC, Raza S, Exarhopoulos A, et al: Urinary metalloproteinases: noninvasive biomarkers for breast cancer risk assessment. Cancer Epidemiol Biomarkers Prev 17:1034-1042, 2008

28. Roy R, Louis G, Loughlin KR, Wiederschain D, Kilroy SM, Lamb CC, et al: Tumor-specific urinary matrix metalloproteinase fingerprinting: identification of high molecular weight urinary matrix metalloproteinase species. Clin Cancer Res 14:6610-6617, 2008

29. Roy R, Yang J, Moses MA: Matrix metalloproteinases as novel biomarkers and potential therapeutic targets in human cancer. J Clin Oncol 27:5287-5297, 2009

30. Roy R, Zurakowski D, Wischhusen J, Frauenhoffer C, Hooshmand S, Kulke M, et al: Urinary TIMP-1 and MMP-2 levels detect the presence of pancreatic malignancies. Br J Cancer 111:1772-1779, 2014

31. Smith ER, Manfredi M, Scott RM, Black PM, Moses MA: A recurrent craniopharyngioma illustrates the potential usefulness of urinary matrix metalloproteinases as noninvasive biomarkers: case report. Neurosurgery 60:E1148-E1149, 2007

32. Smith ER, Zurakowski D, Saad A, Scott RM, Moses MA: Urinary biomarkers predict brain tumor presence and response to therapy. Clin Cancer Res 14:2378-2386, 2008

33. Soutter AD, Nguyen M, Watanabe H, Folkman J: Basic fibroblast growth factor secreted by an animal tumor is detectable in urine. Cancer Res 53:5297-5299, 1993

34. Sreekanthreddy P, Srinivasan H, Kumar DM, Nijaguna MB, Sridevi S, Vrinda M, et al: Identification of potential serum biomarkers of glioblastoma: serum osteopontin levels correlate with poor prognosis. Cancer Epidemiol Biomarkers Prev 19:1409-1422, 2010

35. Takahashi JA, Mori H, Fukumoto M, Igarashi K, Jaye M, Oda Y, et al: Gene expression of fibroblast growth factors in human gliomas and meningiomas: demonstration of cellular source of basic fibroblast growth factor mRNA and peptide in tumor tissues. Proc Natl Acad Sci U S A 87:5710-5714, 1990

36. Zagzag D, Miller DC, Sato Y, Rifkin DB, Burstein DE: Immunohistochemical localization of basic fibroblast growth factor in astrocytomas. Cancer Res 50:7393-7398, 1990

37. Zhou XH, Obuchowski NA, McClish DK: Statistical Methods in Diagnostic Medicine. New York: John Wiley, 2011

\section{Disclosures}

The authors report no conflict of interest concerning the materials or methods used in this study or the findings specified in this paper.

\section{Author Contributions}

Conception and design: Smith, Pricola Fehnel. Acquisition of data: Pricola Fehnel, Duggins-Warf, McKee-Proctor, Majumder, Raber, Han. Analysis and interpretation of data: Smith, Pricola Fehnel. Drafting the article: Pricola Fehnel. Critically revising the article: Smith, Pricola Fehnel. Reviewed submitted version of manuscript: Smith, Pricola Fehnel. Approved the final version of the manuscript on behalf of all authors: Smith. Statistical analysis: Zurakowski. Study supervision: Smith.

\section{Supplemental Information \\ Online-Only Content}

Supplemental material is available with the online version of the article.

Supplementary Tables. http://thejns.org/doi/suppl/10.3171/ 2015.12.PEDS15448.

\section{Previous Presentations}

Portions of this work were presented at the New England Neurosurgical Conference, Brewster, Massachusetts, in June 2014 and in poster form at the CNS Annual Meeting, Boston, Massachusetts, in October 2014.

\section{Correspondence}

Edward R. Smith, Vascular Biology Program, Boston Children's Hospital/Harvard Medical School, 300 Longwood Ave., Boston, MA 02115. email: edward.smith@childrens.harvard.edu. 